\title{
Zusammenhänge zwischen sozialer Unterstützung und Suizidalität bei homo- und bisexuellen Personen
}

Adrian Widmer ${ }^{\mathrm{a}}$, Daniel Reglia ${ }^{\mathrm{a}}$ Lukas Frei $^{\mathrm{a}}$ und Hansjörg Znoja

Übersicht: Der Beitrag untersucht Zusammenhänge zwischen sozialer Unterstützung und Suizidalität bei 615 homo- und bisexuellen Personen in der Schweiz. In einer Internetumfrage wurden dazu Suizidgedanken, Suizidpläne und Suizidversuche, sowie diverse Aspekte sozialer Unterstützung und die soziale Belastung erfragt. Obwohl homo- und bisexuelle Personen hohe Werte sozialer Unterstützung und niedrige Werte sozialer Belastung aufwiesen, zeigten sich hohe 12-Monats- und Lebenszeitprävalenzen bezüglich Suizidgedanken, -plänen und -versuchen. Alle Aspekte sozialer Unterstützung waren mit der Suizidalität in den letzten zwölf Monaten assoziiert, wobei die Zusammenhänge mit der sozialen Integration $(r=-.34)$ und der sozialen Belastung $(r=.39)$ am stärksten ausfielen. Selbst bei Kontrolle der depressiven Stimmung und des Alters teilnehmender Personen blieben die soziale Unterstützung $(\mathrm{OR}=.61)$ und die soziale Belastung $(\mathrm{OR}=2.13)$ prädiktiv für die Suizidalität in den letzten zwölf Monaten. Personen, die viel soziale Unterstützung wahrnahmen (> 1 SD über dem Mittelwert) berichteten fünfmal weniger Suizidalität als solche mit geringer sozialer Unterstützung ( $>1$ SD unter dem Mittelwert). Personen, die viel soziale Belastung in ihrem Umfeld wahrnahmen ( $>1$ SD über dem Mittelwert), zeigten im vergangenen Jahr 14-mal häufiger Suizidalität als Personen, die geringe soziale Belastung erlebten (>1 SD unter dem Mittelwert).

Schlüsselwörter: Bisexualität; Homosexualität; soziale Belastung; soziale Unterstützung; Suizidalität

Sozialer Austausch und das Eingebundensein in einen sozialen Kontext stellen wichtige Voraussetzungen für das psychische und physische Wohlergehen und die Gesundheit einer Person dar (z. B. Fydrich et al. 2007). Im Buch „Why People Die by Suicide“ (Joiner 2005) reflektiert der Autor die Frage, welche Bedingungen erfüllt sein müssen, damit schwere Formen der Selbstverletzung bis hin

a Institut für Psychologie, Universität Bern

Z Sexualforsch 2014; 27; 220-236

(C) Georg Thieme Verlag KG Stuttgart · New York

ISSN 0932-8114

DOI $10.1055 / \mathrm{s}-0034-1385057$ 
zum vollendeten Suizid möglich werden. Er kommt zum Schluss, dass sich Menschen hierzu einerseits durch wiederholte aversive Erfahrungen die Bereitschaft zur schweren Selbstverletzung aneignen und andererseits den Wunsch zum Sterben entwickeln müssen. Letzteres kommt nach Joiner (2005) dann zustande, wenn sich Menschen als nicht-zugehörig oder ihren Mitmenschen gegenüber als Last empfinden. Trotz Verbesserungen in der Stellung homo- und bisexueller Personen in der Schweiz hatten in einer Umfrage in der Westschweiz insgesamt 41.5\% der jungen homo- und bisexuellen Männer den Eindruck, dass die Gesellschaft ihre sexuelle Orientierung nicht wirklich akzeptiere (Cochand und Singy 2001). Internationale Studien belegen, dass homound bisexuelle Personen nach wie vor eine stigmatisierte Minderheit darstellen, die - sowohl in der Gesellschaft als auch in der Familie - häufig Ablehnung und Zurückweisung erleben (z.B. Meyer 2003) oder aufgrund ihrer sexuellen Orientierung gar Freundschaften verloren haben (Diamond und Lucas 2004). Homo- und bisexuelle Personen scheinen aufgrund ihrer sexuellen Orientierung also häufiger die Erfahrung zu machen, sich als nicht-zugehörig und - je nach Situation - ihrem Umfeld gegenüber als Last zu empfinden (vgl. Diamond et al. 2011). Studien konnten bereits einen Zusammenhang zwischen der sozialen Unterstützung und der Suizidalität bei homo- und bisexuellen Personen finden. Diese Arbeit soll dazu dienen, bisherige Ergebnisse zum Zusammenhang von sozialer Unterstützung und Suizidalität bei homo- und bisexuellen Personen zu replizieren und den Zusammenhang verschiedener inhaltlicher Aspekte sozialer Unterstützung mit Suizidalität genauer zu untersuchen.

Diverse Befunde untermauern die Annahme, dass Suizidgedanken, Suizidpläne und Suizidversuche bei homo- und bisexuellen Personen häufiger vorkommen als bei heterosexuellen Personen: In der Metaanalyse von King et al. (2008) zeigten homo- und bisexuelle Personen im Vergleich mit heterosexuellen Personen erhöhte Lebenszeitprävalenzen für Suizidgedanken $(\mathrm{OR}=2.04)$ und Suizidversuche $(\mathrm{OR}=2.47)$. Auch in der Metaanalyse von Marshal et al. (2011) berichteten die Autoren über höhere Raten an Suizidalität (OR=2.92) bei homo- und bisexuellen Jugendlichen verglichen mit heterosexuellen Jugendlichen. Die Unterschiede nahmen mit dem Schweregrad der Suizidalität $\mathrm{zu}$ - von Suizidgedanken ( $\mathrm{OR}=1.96)$, über Suizidpläne $(\mathrm{OR}=2.20)$ und Suizidversuche $(\mathrm{OR}=3.18)$ bis hin zu Suizidversuchen, die medizinische Hilfe notwendig machten $(\mathrm{OR}=4.17)$. In der Studie von Wang et al. (2012) wurden die Ergebnisse von drei zufallsbasierten Stichprobenerhebungen in der Schweiz zusammengefasst: Im Vergleich mit heterosexuellen Jugendlichen und jungen Erwachsenen zeigten homo- und bisexuelle Männer sowohl eine erhöhte Lebenszeitprävalenz für Suizidgedanken $(\mathrm{OR}=2.15)$ und Suizidversuche $(\mathrm{OR} \approx 5)$ als auch eine etwa zweifach erhöhte 12-Monats-Prävalenz für Suizidgedanken, Suizidpläne und Suizidversuche.

Obgleich die erhöhten Prävalenzraten psychischer Störungen und der Suizidalität bei homo- und bisexuellen Personen immer wieder auch die Debatte der Krankheitswertigkeit einer homo- und bisexuellen Orientierung entfachen (z. B. Bailey 1999), werden die erhöhten Prävalenzen im Allgemeinen als Folge von Ablehnung, Vorurteilen, Diskriminierung und Gewalt gegen homo- 
und bisexuelle Personen aufgrund ihrer sexuellen Orientierung erklärt (z.B. Meyer 2003). Im Rahmen des Minoritätenstress-Modells räumte Meyer (2003) der sozialen Unterstützung eine zentrale Rolle hinsichtlich der psychischen Gesundheit von homo- und bisexuellen Personen ein, da sie die negativen Einflüsse unterschiedlicher Stressoren auf die psychische Gesundheit abpuffere. Andere Autoren ergänzten das Minoritätenstress-Modell, indem sie zusätzlich einen direkten Einfluss der sozialen Unterstützung auf die psychische Gesundheit postulierten (Shilo und Savaya 2011; vgl. bereits Cohen und Wills 1985).

Doch was lässt sich genau unter „sozialer Unterstützung“ verstehen? Trotz intensiver Erforschung des Konzeptes besteht unter Forschern nach wie vor wenig Übereinstimmung hinsichtlich einer genauen Definition sowie der operationalen Erfassung sozialer Unterstützung (z. B. Hupcey 1998; Williams et al. 2004). Verschiedene Befunde zeigen aber, dass die psychische Gesundheit insbesondere mit der subjektiven Wahrnehmung sozialer Unterstützung zusammenhängt, während der tatsächlich erfahrenen sozialen Unterstützung eine geringere Bedeutung zukommt (z. B. Wethington und Kessler 1986). Fydrich et al. (2007: 10) umschreiben soziale Unterstützung als „Wahrnehmung und Bewertung von Quantität und Qualität sozialer Beziehungen und Interaktionen“. Damit betonen sie die Wichtigkeit der subjektiven Verarbeitung sozialer Beziehungen und Interaktionen durch das Individuum. Neben der unterstützenden Funktion sozialer Beziehungen sehen diverse Autoren deren Belastungsaspekt als wichtige Ergänzung (z. B. Vinokur und Van Ryn 1993). Teilweise wird die soziale Belastung auch als Teilaspekt sozialer Unterstützung betrachtet (vgl. Shilo und Savaya 2011). Fydrich et al. (2007: 33) verstehen unter der sozialen Belastung „die Wahrnehmung potenziell negativer oder belastender Merkmale bzw. Verhaltensweisen von Personen des sozialen Umfelds“.

Diverse Studien schildern Zusammenhänge zwischen der Wahrnehmung sozialer Unterstützung und Belastung und der Suizidalität homo- und bisexueller Personen. So berichteten in einer Untersuchung von Plöderl und Fartacek (2005) homo- und bisexuelle Erwachsene im Vergleich mit heterosexuellen Erwachsenen über weniger wahrgenommene Unterstützung durch den Vater, die Mutter und die gesamte Familie. Zudem war die wahrgenommene Unterstützung bei homo- und bisexuellen Personen stärker mit der Suizidalität assoziiert als bei heterosexuellen Personen. In einer Studie von Botnick et al. (2002) wiesen homo- und bisexuelle Männer, die einen Suizidversuch begangen hatten, höhere Depressionswerte, weniger soziale Unterstützung, einen niedrigeren Selbstwert und vermehrten Gebrauch von Poppers (Nitritinhalaten) auf. Männer mit geringerer sozialer Unterstützung berichteten doppelt so oft von einem Suizidversuch wie Männer mit großer sozialer Unterstützung. Gerade im Rahmen der Identitätsentwicklung junger homo- und bisexueller Personen scheint der Wahrnehmung sozialer Unterstützung und Belastung eine wichtige Rolle zuzukommen. So zeigten die wahrgenommene soziale Unterstützung und die Akzeptanz durch Freunde und Familie in einer Studie von Shilo und Savaya (2011) positive Effekte auf die Selbstakzeptanz und die Bekanntgabe der sexuellen Orientierung. Umgekehrt wurde die erlebte Ablehnung der sexuellen Orientierung durch die Fa- 
milie von homo- und bisexuellen Jugendlichen mit depressiven und suizidalen Symptomen häufig als Ursache der psychischen Symptome verstanden (Diamond et al. 2011). Die meisten der befragten Jugendlichen wünschten sich außerdem eine engere Beziehung zu ihren Eltern. Erst kürzlich wurden die Risikofaktoren von Suizidgedanken (Liu und Mustanski 2012) und Suizidversuchen (Mustanski und Liu 2013) bei homo-, bi- und transsexuellen Jugendlichen erstmals im Längsschnitt untersucht. Die wahrgenommene Unterstützung durch Freunde und Familie, ebenso wie Viktimisierungserfahrungen waren prädiktiv für aktuelle und zukünftige Suizidgedanken sechs Monate später, unabhängig von Drittfaktoren wie der Impulsivität und bisherigen Suizidversuchen (Liu und Mustanski 2012). Ein etwas anderes Bild zeigte sich im Hinblick auf die Suizidversuche (Mustanski und Liu 2013): Während die depressiven Symptome, die Impulsivität, die Hoffnungslosigkeit, die Viktimisierungserfahrungen und die wahrgenommene familiäre Unterstützung mit den bisherigen Suizidversuchen zusammenhingen, etablierten sich in einem gemeinsamen Modell nur die Hoffnungslosigkeit und die depressiven Symptome als relevante Prädiktoren. Die beiden Mediatoren erklärten den Zusammenhang zwischen familiärer Unterstützung und bisherigen Suizidversuchen. Weder die familiäre Unterstützung noch die Unterstützung durch Freunde zeigten prospektiv einen direkten Zusammenhang mit den Suizidversuchen ein Jahr später.

Während diverse Untersuchungen unterschiedliche Quellen sozialer Unterstützung - insbesondere durch Freunde und durch die Familie - im Hinblick auf die psychische Gesundheit und Suizidalität homo- und bisexueller Personen erforschten, wurden die Zusammenhänge mit inhaltlichen Aspekten sozialer Unterstützung kaum untersucht. Neben der Erhebung deskriptiver Daten zur sozialen Unterstützung und der Suizidalität bei homo- und bisexuellen Personen in der Schweiz bestand das primäre Ziel der vorliegenden Untersuchung darin, die Zusammenhänge zwischen inhaltlichen Aspekten sozialer Unterstützung und der Suizidalität zu untersuchen und dabei bisherige Ergebnisse zum Zusammenhang sozialer Unterstützung und der Suizidalität zu replizieren. Dabei erwarteten wir, dass Aspekte wahrgenommener sozialer Unterstützung negativ und die Wahrnehmung sozialer Belastung positiv mit der Suizidalität in den letzten zwölf Monaten zusammenhängen. Die Berücksichtigung von Drittfaktoren wie Alter und depressive Stimmung sollte zudem zeigen, ob Aspekte sozialer Unterstützung und Belastung unabhängig von diesen Risikofaktoren mit der Suizidalität zusammenhängen.

\section{Methode}

Rekrutierung und Durchführung

Die Datenerhebung erfolgte online. Auf Unipark ${ }^{1}$ erstellten wir dazu einen Fragebogen mit dem Titel „Umfrage zum Wohlbefinden sexueller Minderheiten“,

1 URL: www.unipark.de. 
der vor der Erhebung von der Ethikkommission der Universität Bern geprüft und genehmigt wurde. Die Umfrage richtete sich an Personen, die sich sexuell zu gleichgeschlechtlichen Personen hingezogen fühlten, sich als lesbisch, schwul oder bisexuell identifizierten oder bereits gleichgeschlechtlichen Sexualkontakt hatten. Außerdem mussten die teilnehmenden Personen mindestens 16 Jahre alt sein und zum Zeitpunkt der Erhebung in der Schweiz wohnen. Die Rekrutierung der Versuchspersonen erfolgte per „Schneeballsystem“: Dazu wurden zahlreiche Gruppen und Organisationen für homo- und bisexuelle Personen gebeten, den Link zur Umfrage an ihre Mitglieder weiterzuleiten. Zusätzlich wurden Personen aus dem Bekanntenkreis angefragt. Auf Purplemoon ${ }^{2}$ - einer Kommunikations- und Kontaktplattform für homo- und bisexuelle Personen - wurde zudem eine Werbeanzeige platziert. Das Ausfüllen des Fragebogens dauerte zwischen 20 und 25 Minuten und erfolgte im Forced-Choice Antwortformat. Die Datenerhebung startete Anfang Dezember 2012 und dauerte bis Ende Januar 2013.

\section{Versuchspersonen}

Der Fragebogen wurde während der Datenerhebung 1'494 Male aufgerufen. Insgesamt wurde die Umfrage von 898 Personen begonnen und von 642 Personen abgeschlossen. Davon wurden 23 Personen aufgrund ihrer Geschlechtsangabe als inter- oder transsexuell, drei Personen aufgrund ihres Wohnortes außerhalb der Schweiz und eine weitere Person aufgrund ihres Antwortmusters ausgeschlossen. Die endgültige Stichprobe setzte sich aus 615 Personen zusammen, darunter 326 Männer und 289 Frauen. Die teilnehmenden Personen waren zum Erhebungszeitpunkt zwischen 16 und 84 Jahre alt $(M=30.33$, $\mathrm{SD}=11.56, \mathrm{MD}=27)$ und in 22 der 26 Kantone der Schweiz wohnhaft. Der größte Anteil stammte aus den Kantonen Zürich (157 Personen) und Bern (133 Personen). 338 Personen wurden durch die Anzeige auf Purplemoon, 65 Personen durch die schweizerische Schwulen- und Lesbenorganisation (Pink Cross und LOS), 89 Personen durch die Anfrage an weitere Gruppen für homound bisexuelle Personen und 121 Personen durch ihren Bekanntenkreis auf die Umfrage aufmerksam gemacht. Bei zwei Personen konnte nicht klar nachvollzogen werden, wie sie von der Umfrage erfahren hatten. 331 Personen waren zum Zeitpunkt der Umfrage Single, 245 Personen in einer Beziehung, 33 Personen lebten in einer registrierten Partnerschaft und sechs Personen waren verheiratet. 156 Personen verfügten über einen Hochschulabschluss, 73 Personen über eine abgeschlossene höhere Berufsausbildung. 142 Personen nannten die Maturität, 171 Personen eine abgeschlossene Berufslehre und 73 Personen einen Primar- oder Sekundarschulabschluss als höchste abgeschlossene Ausbildung. Zum Erhebungszeitpunkt waren 406 Personen berufstätig, 140 Personen studierten und 34 Personen gingen zur Schule. Zwei waren

2 URL: www.purplemoon.ch. 
Hausfrau bzw. Hausmann. 22 Personen waren zum Zeitpunkt der Erhebung arbeitslos und elf Personen pensioniert.

\section{Sexuelle Orientierung}

Zur Kategorisierung von Homo- und Bisexualität verwendeten wir die Dimensionen der sexuellen Anziehung, der Selbstidentifikation und des Sexualverhaltens. Personen wurden als homo- und bisexuell kategorisiert, wenn sie sich sexuell zumindest teilweise von gleichgeschlechtlichen Personen angezogen fühlten und/oder sich als lesbisch, schwul oder bisexuell bezeichneten und/oder bereits gleichgeschlechtlichen Sexualkontakt hatten. In unserer Stichprobe vermerkten 366 Personen ausschließlich gleichgeschlechtliche sexuelle Anziehung und 245 Personen zumindest teilweise gleich- und gegengeschlechtliche sexuelle Anziehung. Vier Personen gaben an, sich im Hinblick auf ihre sexuelle Anziehung unsicher zu sein. 492 Personen bezeichneten sich als homosexuell, 99 Personen als bisexuell (einschließlich omni- und pansexuell) und drei Personen als heterosexuell. Zehn Personen wussten es nicht und elf Personen konnten aufgrund ihrer Angabe keiner der bisherigen Kategorien zugeordnet werden. Bezogen auf ihr bisheriges Leben berichteten 256 der befragten Personen über ausschließlich gleichgeschlechtlichen Sex, 328 Personen hatten sowohl gleich- als auch gegengeschlechtlichen Sex, 17 Personen ausschließlich gegengeschlechtlichen Sex. 14 Personen gaben an, keinen Sex gehabt zu haben.

\section{Soziale Unterstützung}

Zur Erfassung der sozialen Unterstützung verwendeten wir den „Fragebogen zur sozialen Unterstützung“ (F-SozU; Sommer und Fydrich 1991; Fydrich et al. 2007) der bereits in zahlreichen Studien zur Untersuchung der psychischen Gesundheit und Belastung, den Auswirkungen somatischer Erkrankungen und der Therapieevaluation eingesetzt wurde (z. B. Förtsch et al. 2003; Franke et al. 2003; Krampe et al. 2003). Der Fragebogen erfasst die subjektiv wahrgenommene Form sozialer Unterstützung. Er beschreibt damit die Überzeugung einer Person, in verschiedenen Bereichen durch wichtige Menschen wie den Partner oder die Partnerin, Familienmitglieder, Freunde, Bekannte oder Nachbarn unterstützt zu werden oder bei Bedarf Hilfe aus dem sozialen Umfeld zu erhalten (Fydrich et al. 2007). Erhoben wurden die Skalen Emotionale Unterstützung, Praktische Unterstützung, Soziale Integration und Soziale Belastung (Cronbach's Alpha bezieht sich jeweils auf die erhobene Stichprobe): Die Skala Emotionale Unterstützung erfasst nach Fydrich et al. (2007) die Wahrnehmung, von anderen Personen gemocht und akzeptiert zu werden, die eigenen Gefühle mitteilen zu können und Anteilnahme zu erleben (16 Items, $\alpha=.92$, z. B. „Es gibt Menschen, die Freude und Leid mit mir teilen.“) Die Skala Praktische Unterstützung beschreibt die Wahrnehmung, praktische Hilfe bei alltäglichen Problemen zu erhalten (9 Items, $\alpha=.87$, z. B. „Wenn ich wirklich mal unter Stress stehe, werden mir auch mal 
Aufgaben abgenommen.“) Inhalt der Skala Soziale Integration ist die wahrgenommene Zugehörigkeit zu einem Freundeskreis mit ähnlichen Wertvorstellungen und Interessen (13 Items, $\alpha=.89$, z. B. „Viele meiner Freunde/Angehörigen haben eine ähnliche Einstellung zum Leben wie ich.") Mit der Skala Soziale Belastung schließlich wird nach Fydrich et al. (2007) die Wahrnehmung negativer oder belastender Merkmale von Personen aus dem sozialen Umfeld erhoben (12 Items, $\alpha=.87$, z. B. „Ich fühle mich von wichtigen Personen abgelehnt.“) Mit den Items der Skalen Emotionale Unterstützung, Praktische Unterstützung und Soziale Integration, lässt sich zudem die Gesamtskala Wahrgenommene soziale Unterstützung (38 Items, $\alpha=.96$ ) bilden. Die teilnehmenden Personen mussten die insgesamt 50 Items auf einer fünfstufigen Skala beurteilen (,trifft nicht zu“, „trifft eher nicht zu“, „trifft teilweise zu“, „trifft zu“, „trifft genau zu“). Nach dem Umcodieren invertierter Items (10 Items) wurde für jede Skala ein Mittelwert gebildet, wobei höhere Werte mehr soziale Unterstützung oder Belastung bedeuten. Die verwendeten Skalen des F-SozU korrelierten hoch untereinander $(|\mathrm{r}|<.78$, s. Tab.1) und übertrafen die von Fydrich et al. (1999) genannten Werte $(|r|<.71)$ teilweise deutlich. Zur Analyse der Skalenstruktur wurde eine explorative Faktorenanalyse mit orthogonaler Rotation (Varimax) durchgeführt, welche die postulierten Originalskalen allerdings nicht replizieren konnte: Insgesamt wiesen sieben Faktoren einen Eigenwert $>1$ auf und erklärten zusammen 57.52 \% der Varianz. Der Scree-Plot wies allerdings auf eine zwei-FaktorenLösung hin. Daher wurde eine zweite Faktorenanalyse mit orthogonaler Rotation (Varimax) mit zwei zu extrahierenden Faktoren durchgeführt. Die beiden Faktoren erklärten $43.39 \%$ der Varianz $(\mathrm{KMO}=.97$, Bartlett-Test der Sphärizität $X^{2}(1225)=16$ '821.69, $\left.\mathrm{p}<.001\right)$ und passten weitgehend mit den von Fydrich et al. (2007) postulierten Skalen Wahrgenommene soziale Unterstützung und Soziale Belastung überein: Alle Items der Skala Praktische Unterstützung und der größte Teil der Items der Skalen Emotionale Unterstützung und Soziale Integration korrelierten am stärksten mit dem ersten Faktor. Alle Items der Skala Soziale Belastung, ebenso wie ein Item der Skala Emotionale Unterstützung und fünf Items der Skala Soziale Integration wiesen die stärkste Ladung auf dem zweiten Faktor auf.

Tab. 1 Bivariate Korrelationen der Skalen sozialer Unterstützung und sozialer Belastung ( $N=615)$.

\begin{tabular}{llllll}
\hline & EU & PU & SI & Bel & Cronbach's Alpha \\
\hline EU & & & & & .92 \\
PU & $.77^{* * *}$ & & & .87 \\
SI & $.77^{* * *}$ & $.71^{* * *}$ & & .89 \\
Bel & $-.55^{* * *}$ & $-.52^{* * *}$ & $-.66^{* * *}$ & & .87 \\
WasU & $.94^{* * *}$ & $.88^{* * *}$ & $.91^{* * *}$ & $-.64^{* * *}$ & .96 \\
\hline
\end{tabular}

Skalen: $\mathrm{EU}=$ Emotionale Unterstützung, $\mathrm{PU}$ = Praktische Unterstützung, $\mathrm{SI}$ = Soziale Integration, Bel = Soziale Belastung, WasU $=$ Wahrgenommene soziale Unterstützung; ${ }^{* * *} \mathrm{p}<.001$. 


\section{Depressive Stimmung}

Um verschiedene Aspekte des Wohlbefindens zu erheben, wurde der „Berner Fragebogen zum Wohlbefinden für Erwachsene“ (BFW/E) verwendet (Grob 1995). Die Skala Depressive Stimmung wurde dabei mit fünf Items erfasst ( $\alpha=.85$, z. B. „Nichts macht mir mehr richtig Freude.“). Die Beurteilung der Aussagen erfolgte anhand einer sechsstufigen Skala („ist total falsch“, „ist sehr falsch“, „ist eher falsch“, „ist eher richtig“, „ist sehr richtig“, „ist total richtig“). Auch hier wurde ein Skalenmittelwert errechnet, wobei höhere Werte eine stärker ausgeprägte depressive Stimmung implizieren.

\section{Suizidalität}

Zur Erfassung der Suizidalität verwendeten wir die deutsche Übersetzung (Nindl 2001) der Fragen von Paykel et al. (1974). Die Fragen deckten das Kontinuum von Suizidgedanken („Haben Sie jemals daran gedacht, sich Ihr Leben zu nehmen, auch wenn Sie es gar nicht wirklich tun wollten?“) über Suizidpläne („Haben Sie jemals den Punkt erreicht, an dem Sie ernsthaft überlegt haben, sich umzubringen oder vielleicht Pläne gemacht haben, wie Sie es machen würden?“) bis zu Suizidversuchen („Haben Sie jemals einen Suizidversuch gemacht?") ab. Für jede der drei Fragen standen folgende Antwortkategorien zur Auswahl: „Ja, innerhalb der letzten 12 Monate“, „Ja, aber davor“, „nein, nie“. Dadurch konnte für jede Form der Suizidalität (Suizidgedanken, -pläne, -versuche) sowohl eine dichotome 12-MonatsPrävalenz als auch Lebenszeitprävalenz errechnet werden.

\section{Statistische Auswertungen}

Alle statistischen Berechnungen wurden mit SPSS (Release 20.0) für Macintosh durchgeführt. Zur Berechnung von Zusammenhängen wurden bivariate Korrelationen nach Pearson verwendet. Die Vorhersage der Suizidalität in den letzten zwölf Monaten erfolgte in einer logistischen Regression.

\section{Ergebnisse}

Für alle Analysen wurde ein $\alpha$-Level von .05 festgelegt. In Tab. 2 sind die Mittelwerte der Skalen sozialer Unterstützung und Belastung getrennt nach Geschlecht dargestellt. Insgesamt wurden hohe Werte sozialer Unterstützung und niedrige Werte sozialer Belastung erreicht. Das Geschlecht zeigte signifikante Zusammenhänge mit den Skalen Emotionale Unterstützung $(r=.20, p<.001)$, Praktische Unterstützung $(r=.13, p<.01)$, Soziale Integration $(r=.15, p<.001)$ und der Gesamtskala Wahrgenommene soziale Unterstützung $(r=.18, p<.001)$. Frauen erreichten insgesamt leicht höhere Werte sozialer Unterstützung als Männer. Keinen Zusammenhang zeigte das Geschlecht mit der Skala Soziale Belastung $(r=-.04, p=.33)$. Das Alter 
Tab. 2 Mittelwerte (und Standardabweichungen) von Aspekten sozialer Unterstützung und Belastung getrennt nach Geschlecht.

\begin{tabular}{lllllll}
\hline & $\boldsymbol{n}$ & EU & PU & SI & Bel & WasU \\
\hline Total & 615 & $4.08(.71)$ & $3.76(.74)$ & $3.61(.74)$ & $2.07(.69)$ & $3.85(.67)$ \\
Männer & 326 & $3.95(.77)$ & $3.67(.78)$ & $3.51(.77)$ & $2.10(.72)$ & $3.73(.71)$ \\
Frauen & 289 & $4.23(.60)$ & $3.87(.68)$ & $3.73(.68)$ & $2.04(.64)$ & $3.97(.59)$ \\
\hline
\end{tabular}

Skalen: EU = Emotionale Unterstützung, PU = Praktische Unterstützung, SI = Soziale Integration, Bel = Soziale Belastung, WasU $=$ Wahrgenommene soziale Unterstützung.

zeigte signifikant negative Zusammenhänge mit den Skalen Emotionale Unterstützung ( $r=-.08, p<.05)$, Praktische Unterstützung $(r=-.12, p<.01)$ und Soziale Belastung ( $r=-.16, p<.001$ ), während die Zusammenhänge mit der Skala Soziale Integration und der Gesamtskala Wahrgenommene soziale Unterstützung nicht signifikant ausfielen $(|r|<-.06, p>.18)$. Die sexuelle Orientierung (homosexuell vs. bisexuell) zeigte einen signifikanten Zusammenhang mit der Skala Soziale Belastung, allerdings nur, wenn die Kategorisierung der sexuellen Orientierung anhand der sexuellen Anziehung $\left(\mathrm{n}_{\text {homo }}=366, \mathrm{n}_{\mathrm{bi}}=245, r=.11, p<.01\right)$ oder der Selbstidentifikation $\left(\mathrm{n}_{\text {homo }}=\right.$ $492, \mathrm{n}_{\mathrm{bi}}=99, r=.11, p<.01$ ) erfolgte. Bisexuelle Personen wiesen insgesamt leicht höhere Werte sozialer Belastung auf als homosexuelle Personen. Keinen Zusammenhang zeigte die sexuelle Orientierung mit den Skalen Emotionale Unterstützung, Praktische Unterstützung, Soziale Integration und der Gesamtskala Wahrgenommene soziale Unterstützung $(|r|<.07, p>.10)$.

Für den Zeitraum der vergangenen zwölf Monate berichteten 104 Personen (16.9\%) von Suizidgedanken, 56 Personen (9.1\%) von Suizidplänen und 14 Personen ( $2.3 \%$ ) von einem Suizidversuch. Im Hinblick auf ihr bisheriges Leben vermerkten 400 Personen (65\%) Suizidgedanken, 269 Personen (43.7\%) Suizidpläne und 84 Personen (13.7\%) einen Suizidversuch. Das Geschlecht wies keinen signifikanten Zusammenhang zu den einzelnen Suizidalitätsmaßen auf $(|r|<.04, p>.40)$, während das Alter signifikant negativ mit den Suizidgedanken $(r=-.23, p<.001)$, Suizidplänen $(r=-.20, p<.001)$ und Suizidversuchen $(r=-.10, p<.05)$ der letzten zwölf Monate korrelierte. Im Hinblick auf das bisherige Leben fand sich allerdings nur ein signifikanter Zusammenhang zwischen dem Alter der teilnehmenden Personen und den bisherigen Suizidgedanken $(r=-.14, \mathrm{p}<.01)$. Tab. 3 zeigt die Prävalenzen von Suizidgedanken, -plänen, und -versuchen der letzten zwölf Monate für unterschiedliche Altersgruppen. In Frei et al. (2013) sind zusätzlich die 12-Monats- und Lebenszeitprävalenzen von Suizidgedanken, Suizidplänen und Suizidversuchen getrennt nach Geschlecht dargestellt. Die sexuelle Orientierung zeigte einen signifikanten Zusammenhang mit den bisherigen Suizidplänen $\left(\mathrm{n}_{\text {homo }}=256, \mathrm{n}_{\mathrm{bi}}=328, r=.10, p<.05\right)$ und Suizidversuchen $\left(\mathrm{n}_{\text {homo }}=256, \mathrm{n}_{\mathrm{bi}}=328, r=.11, p<.01\right)$, wenn die Kategorisierung der sexuellen Orientierung anhand des bisherigen Sexualverhaltens erfolgte: Bisexuelle Personen berichteten dabei insgesamt etwas häufiger über Suizidpläne und Suizidversuche im bisherigen Leben. Keinen Zusammenhang zeigte die 
Tab. 3 Prävalenzen (und 95\%-Konfidenzintervalle) von Suizidgedanken, -plänen und -versuchen während der letzten zwölf Monate bei verschiedenen Altersgruppen. *

\begin{tabular}{lcll}
\hline Alter & Suizidgedanken & Suizidpläne & Suizidversuche \\
\hline 16 - 20 Jahre $(n=114)$ & $30.7(22.8-39.5)$ & $18.4(11.4-25.4)$ & $3.5(0.9-7.0)$ \\
21 - 25 Jahre $(n=169)$ & $20.7(14.8-26.6)$ & $12.4(7.7-17.8)$ & $4.1(1.2-7.1)$ \\
26 - 30 Jahre $(n=97)$ & $13.4(6.2-20.6)$ & $7.2(2.1-12.4)$ & $2.1(0.0-5.2)$ \\
31 - 40 Jahre $(n=119)$ & $14.3(8.4-21.0)$ & $5.9(2.5-10.1)$ & 0.0 \\
$>$ 40 Jahre $(n=116)$ & $3.4(0.9-6.9)$ & 0.0 & $0.9(0.0-2.6)$ \\
Total $(n=615)$ & $16.9(14.1-20.0)$ & $9.1(7.0-11.4)$ & $2.3(1.1-3.6)$ \\
\hline
\end{tabular}

${ }^{*}$ Vgl. Frei et al. 2013.

sexuelle Orientierung allerdings mit den bisherigen Suizidgedanken und den Suizidgedanken, -plänen und -versuchen in den letzten zwölf Monaten $(|r|<.05, \mathrm{p}>.25)$.

Zur Berechnung der Zusammenhänge verschiedener Aspekte sozialer Unterstützung und Suizidalität in den letzten zwölf Monaten wurden die verschiedenen Suizidalitätsmaße (Suizidgedanken, -pläne und -versuche) der letzten zwölf Monate in einer dichotomen Variable zusammengefasst. Personen, die in der Umfrage entweder Suizidgedanken, Suizidpläne oder Suizidversuche angegeben hatten, erhielten den Wert 1 und Personen, die über keine Form der Suizidalität berichtet hatten, den Wert 0. Im Hinblick auf die letzten zwölf Monate zeigten 111 Personen (18\%) mindestens eine Form der Suizidalität, während 504 Personen (82\%) über keine Form der Suizidalität berichteten. Alle Aspekte sozialer Unterstützung zeigten einen signifikanten Zusammenhang mit der Suizidalität in den vergangenen zwölf Monaten: Während die Skalen Emotionale Unterstützung ( $r=-.27$, $p<.001)$, Praktische Unterstützung $(r=-.22, p<.001)$, Soziale Integration $(r=-.34, p<.001)$ und die Gesamtskala Wahrgenommene soziale Unterstützung $(r=-.31, p<.001)$ signifikant negativ mit der Suizidalität in den letzten zwölf Monaten korrelierten, zeigte die Skala Soziale Belastung $(r=.39$, $p<.001$ ) eine signifikant positive Korrelation. Aufgrund der hohen Interkorrelationen der Skalen sozialer Unterstützung und der besseren Passung mit der faktorenanalytisch eruierten Skalenstruktur verwendeten wir für die weiteren Analysen nur noch die Gesamtskala Wahrgenommene soziale Unterstützung, die sich aus den Skalen Emotionale Unterstützung, Praktische Unterstützung und Soziale Integration zusammensetzen lässt, sowie die Skala Soziale Belastung nach Fydrich et al. (2007).

In einer logistischen Regression wurden die Suizidalität in den letzten zwölf Monaten als abhängige Variable und die Wahrgenommene soziale Unterstützung, die Soziale Belastung, die Depressive Stimmung und das Alter der teilnehmenden Personen als Prädiktoren festgelegt (s. Tab.4). Letztere erklärten 21\% (Cox \& Snell) respektive 35\% (Nagelkerke) der Varianz $\left(X^{2}(4)=145.70, p<.001\right)$. Sowohl die Wahrgenommene soziale Unterstützung $\left(b=-.50\right.$, Wald $\left.x^{2}(1), p<.05\right)$, die Soziale Belastung $\left(b=.75\right.$, Wald $x^{2}(1)$, 
Tab. 4 Prädiktoren für Suizidalität während der letzten zwölf Monate in einer logistischen Regression ( $\mathrm{N}=615)$.

\begin{tabular}{llll}
\hline & B (SE) & Odds Ratio & 95\%-Konfidenzintervall \\
\hline Bel & $.75(.23)^{* *}$ & 2.13 & $1.36-3.33$ \\
WasU & $-.50(.25)^{*}$ & .61 & $.38-.98$ \\
Dep & $.48(.14)^{* *}$ & 1.61 & $1.22-2.13$ \\
Alter & $-.09(.02)^{* * *}$ & .91 & $.88-.95$ \\
Konstante & $-.17(1.53)$ & & \\
\hline
\end{tabular}

Skalen: $\mathrm{Bel}=$ Soziale Belastung, $\mathrm{WasU}=$ Wahrgenommene soziale Unterstützung, Dep = Depressive Stimmung;

$R^{2}=.21$ (Cox \& Snell), . 35 (Nagelkerke), Modell $X^{2}(4)=145.70, p<.001 .{ }^{*} p<.05,{ }^{* *} p<.01,{ }^{* * *} p<.001$.

$p<.01$ ), und die Depressive Stimmung ( $b=.48$, Wald $\left.\chi^{2}(1), p<.01\right)$ als auch das Alter $\left(b=-.09\right.$, Wald $\left.X^{2}(1), p<.001\right)$ resultierten als signifikante Prädiktoren. Die Odds Ratio für die Gesamtskala Wahrgenommene soziale Unterstützung betrug .61, für die Soziale Belastung 2.13. Bei der Zunahme sozialer Unterstützung um eine Einheit reduzierte sich die Wahrscheinlichkeit für Suizidalität knapp um die Hälfte; bei der Zunahme sozialer Belastung um eine Einheit verdoppelte sich die Wahrscheinlichkeit für Suizidalität.

Während 8.2\% der Personen mit großer sozialer Unterstützung (>1 SD über dem Mittelwert) von Suizidalität im vergangenen Jahr berichteten, stieg dieser Anteil bei Personen mit geringer sozialer Unterstützung (>1 SD unter dem Mittelwert) auf $42.6 \%$. Von den Personen mit geringer sozialer Belastung (>1 SD unter dem Mittelwert) berichteten 2.9\% Suizidalität im vergangenen Jahr, während insgesamt 40.8 \% der Personen mit großer sozialer Belastung (>1 SD über dem Mittelwert) von Suizidalität berichteten.

\section{Diskussion}

Neben der Erhebung der Suizidalität und der sozialen Unterstützung bei homo- und bisexuellen Personen in der Schweiz untersuchte unsere Studie die Zusammenhänge von inhaltlichen Aspekten sozialer Unterstützung und Suizidalität in den letzten zwölf Monaten. Dabei zeigten sich folgende relevante Ergebnisse: Homo- und bisexuelle Personen wiesen hohe Werte sozialer Unterstützung und niedrige Werte sozialer Belastung auf. Dabei unterschieden sich die gefundenen Werte kaum von denen der Normierungsstichprobe (Fydrich et al. 1999). Insbesondere bei jungen Personen zeigten sich hohe Prävalenzen an Suizidgedanken, Suizidplänen und Suizidversuchen. Alle Aspekte sozialer Unterstützung und sozialer Belastung waren mit der Suizidalität in den letzten zwölf Monaten assoziiert. Selbst bei simultaner Berücksichtigung und unter Kontrolle des Alters und der depressiven Stimmung waren sowohl die soziale Unterstützung als auch die soziale Belastung prädiktiv für die Suizidalität in den letzten zwölf Monaten. 
Im Unterschied zu Untersuchungen bei homo- und bisexuellen Jugendlichen (z. B. Shilo und Savaya 2011) hing das Geschlecht in unserer Untersuchung mit den verschiedenen Aspekten sozialer Unterstützung zusammen: Homo- und bisexuelle Frauen wiesen leicht höhere Werte sozialer Unterstützung auf als homo- und bisexuelle Männer. Die Effekte waren allerdings klein, insgesamt aber vergleichbar mit den Befunden der Normierungsstichprobe des F-SozU (Fydrich et al. 2007). Die leichte Abnahme sozialer Unterstützung und Belastung mit zunehmendem Alter ist ebenfalls vergleichbar mit den Befunden der Normierungsstichprobe.

Die hohen Prävalenzraten an Suizidgedanken, -plänen und -versuchen stimmen mit internationalen und nationalen Untersuchungen überein, die zeigen, dass Suizidalität bei homo- und bisexuellen Personen vergleichsweise häufig vorkommt (vgl. King et al. 2008; Marshal et al. 2011; Wang et al. 2012). Bezogen auf ihr bisheriges Leben zeigten in unserer Studie 65\% der teilnehmenden Personen Suizidgedanken, 43.7\% Suizidpläne und 13.7\% verübten einen Suizidversuch. Für die letzten zwölf Monate berichteten 16.9\% über Suizidgedanken, 9.1\% über Suizidpläne, $2.3 \%$ von einem Suizidversuch. Jüngere Personen (16 bis 20 Jahre) zeigten für das vergangene Jahr die höchsten Prävalenzen. Diese Ergebnisse lassen sich mit einer repräsentativen Erhebung unter 16- bis 20-jährigen SchülerInnen und GymnasiastInnen aus dem Jahre 2002 in der Schweiz vergleichen (Wang et al. 2012). Im Hinblick auf das vergangene Jahr berichteten darin $29.2 \%$ der homo- und bisexuellen jungen Männer über Suizidgedanken und 3.1\% von einem Suizidversuch. Die Suizidpläne fielen in dieser Stichprobe mit 30.3\% aber deutlich höher aus als in unserer Studie. In einer zufallsbasierten Erhebung, die 2002 unter homo- und bisexuellen Männern aus Genf durchgeführt wurde (Wang et al. 2012), wiesen homo- und bisexuelle Männern im Alter zwischen 16 und 20 Jahren allerdings noch höhere Prävalenzen auf - insbesondere bei den Suizidgedanken und Suizidversuchen: Für das vergangene Jahr berichteten darin 48.8 \% der jungen Männer über Suizidgedanken, 25 \% über Suizidpläne und $17.1 \%$ hatten einen Suizidversuch unternommen. Die Rekrutierung erfolgte in dieser Erhebung bei Veranstaltungen und Partys, in Bars, Cafés, Discos, Sex-Clubs, Saunen, Parks, Toiletten und Internet-Chaträumen, die primär von homo- und bisexuellen Männern frequentiert wurden (vgl. Wang et al. 2007). Hinsichtlich dieser Studie merkten Wang et al. (2012) an, dass durch die verwendete Rekrutierungsart vorwiegend junge Männer erfasst wurden, deren Meilensteine der sexuellen Identitätsentwicklung vergleichsweise früh erreicht wurden: Diverse Studien fanden einen entsprechenden Zusammenhang zwischen früher homosexueller Identitätsentwicklung und erhöhtem sozialen und psychischen Stress (z. B. Friedman et al. 2008), was nach Wang et al. (2012) die erhöhten Suizidalitätsprävalenzen in dieser Stichprobe zumindest teilweise erklären könnte.

In unserer Untersuchung wurde ein Zusammenhang von allen Aspekten sozialer Unterstützung und Belastung mit der Suizidalität gefunden. Personen, die sich emotional und in praktischen Alltagsangelegenheiten als weniger unterstützt erlebten, sich weniger zu einem Freundeskreis zugehörig 
fühlten und mehr Belastung im sozialen Umfeld wahrnahmen, wiesen vermehrt Suizidalität in den letzten zwölf Monaten auf. Die stärksten Zusammenhänge zeigten sich insbesondere mit der sozialen Belastung und der sozialen Integration, während die emotionale Unterstützung und die praktische Unterstützung weniger stark mit der Suizidalität in den letzten zwölf Monaten zusammenhingen.

Obwohl die soziale Unterstützung und die soziale Belastung nicht unabhängig voneinander waren, blieben beide Aspekte bei simultaner Berücksichtigung prädiktiv für die Suizidalität in den letzten zwölf Monaten. Sowohl der Belastung als auch der Unterstützung durch das soziale Umfeld scheint also im Hinblick auf die Suizidalität eine wichtige Rolle zuzukommen. Obwohl in einigen der präsentierten Modelle (z. B. Shilo und Savaya 2011) eine kausale Wirkung sozialer Unterstützung und sozialer Belastung auf die psychische Gesundheit postuliert wurde, kann die Frage der Kausalität auf der Basis nicht-experimenteller Studien nicht abschließend geklärt werden. Auch unsere Studie kann aufgrund des Querschnittdesigns keine Aussage zur zugrundeliegenden Kausalität geben. Trotz einiger Hinweise zur relativen Stabilität sozialer Unterstützung während einer Woche $(r=.96$; Fydrich et al. 2009), respektive während zweier Monate ( $r=.65$; Fydrich et al. 2007) stellt sich aufgrund der retrospektiven Erhebung der Suizidalität die Frage, ob nicht die Suizidalität das Ausmaß der wahrgenommenen sozialen Unterstützung und Belastung zumindest teilweise mitverursacht haben könnte. So beschreibt Joiner (2005), dass sich suizidale Personen subjektiv als stärker sozial isoliert wahrnehmen und sich ihrem Umfeld gegenüber verstärkt als Last empfinden. Diese Einschätzung entspricht allerdings oftmals nicht der objektiven Realität, vielmehr spiegelt sie ein verzerrtes Wahrnehmen sozialer Kontakte wider (vgl. Joiner 2005). Es wäre also auch denkbar, dass eine verzerrte Wahrnehmung sozialer Kontakte als Folge der Suizidalität die Beurteilung sozialer Unterstützung und Belastung beeinflusste. Trotzdem wird die Annahme, dass ein Mangel sozialer Unterstützung auch kausal bei der Entstehung von Suizidalität und im Speziellen der Entstehung von Suizidgedanken beteiligt sein könnte, durch die Längsschnittstudie von Liu und Mustanski (2012) gestützt, in der die wahrgenommene soziale Unterstützung prädiktiv für aktuelle und zukünftige Suizidgedanken sechs Monate später war. Während in der Studie von Mustanski und Liu (2013) der Zusammenhang zwischen sozialer Unterstützung und vergangenen Suizidversuchen vollständig durch depressive Symptome und Hoffnungslosigkeit erklärt werden konnte, blieben in unserer Studie die soziale Unterstützung und die soziale Belastung selbst bei Kontrolle des Alters und der depressiven Stimmung prädiktiv für die Suizidalität in den letzten zwölf Monaten. Obwohl dieses Ergebnis nicht ausschließen kann, dass die Assoziation durch nicht berücksichtigte weitere Drittvariablen erklärt werden kann, zeigt es dennoch, dass soziale Unterstützung und Belastung unabhängig von wichtigen Risikofaktoren mit der Suizidalität zusammenhängen. Die Bedeutung sozialer Unterstützung und Belastung im Hinblick auf Suizidalität wird beim Vergleich von Personen mit großen vs. geringen sozialen Ressourcen 
zusätzlich unterstrichen. Diese Ergebnisse haben praktische Relevanz für die klinische Arbeit mit homo- und bisexuellen Personen und geben darüber hinaus Hinweise für die Suizidalitäts- und Suizidprävention in dieser Gruppe. Die Resultate unterstreichen dabei die Wichtigkeit, soziale Ressourcen bei homo- und bisexuellen Personen zu fördern und soziale Belastungsfaktoren im sozialen Umfeld zu reduzieren. In diesem Zusammenhang dürfte weitere Aufklärungsarbeit und eine Steigerung der Akzeptanz einer homo- und bisexuellen Orientierung in der Allgemeinbevölkerung nicht unwesentlich sein.

Auf einige wichtige Einschränkungen der Studie soll im Folgenden eingegangen werden. Die Rekrutierung der Versuchspersonen erfolgte nicht durch eine randomisierte Auswahl. Meyer und Wilson (2009) verdeutlichten, dass eine randomisierte Stichprobe insbesondere dann unverzichtbar ist, wenn Populationsparameter geschätzt werden sollen. In diesem Sinne ist u. a. im Hinblick auf die eruierten Prävalenzraten von Suizidgedanken, -plänen und -versuchen Vorsicht geboten. Wie bereits beschrieben, lassen sich die gefundenen Prävalenzen aber durchaus mit einer repräsentativen Erhebung aus der Schweiz vergleichen (Wang et al. 2012). Darüber hinaus bleiben im Hinblick auf die verwendete Rekrutierungsart zwei wichtige Tatsachen zu bedenken: Erstens beschränkte sich unsere Studie auf homo- und bisexuelle Personen, die mindestens 16 Jahre alt waren. Der Median für den ersten Suizidversuch lag in unserer Stichprobe ebenfalls bei 16 Jahren. Dies gibt einen Hinweis darauf, dass unsere Stichprobe einen wesentlichen Altersbereich vernachlässigt, in dem Suizidversuche im Zusammenhang mit dem Finden der eigenen Identität und dem Coming-out häufig vorkommen. Zweitens ist denkbar, dass die gewählte Rekrutierungsart über soziale Netzwerke, Gruppen für homo- und bisexuelle Personen oder über den Bekanntenkreis systematisch Personen vernachlässigte, denen diese sozialen Ressourcen nicht zur Verfügung stehen und die womöglich ein erhöhtes Risiko für Suizidalität aufweisen.

Homo- und bisexuelle Personen wurden in dieser Untersuchung in einer Kategorie zusammengefasst. Tatsächlich bilden homo- und bisexuelle Personen aber keine homogene Gruppe (z. B. Meyer 2003) und auch in unserer Untersuchung berichteten bisexuelle Personen je nach Art der Kategorisierung häufiger über Suizidpläne und Suizidversuche in ihrem bisherigen Leben und zeigten darüber hinaus eine größere soziale Belastung als homosexuelle Personen. Obwohl diese Effekte klein waren und sich nicht mit jeder Kategorisierungsform hinsichtlich der sexuellen Orientierung ergaben, stimmen sie in ihrer Tendenz mit den Ergebnissen anderer Studien überein, die zeigen, dass bisexuelle Personen ein zusätzlich erhöhtes Risiko für Suizidalität aufweisen (z. B. Marshal et al. 2011). Eine weitere Einschränkung rührt daher, dass die Suizidalität ausschließlich für den Zeitraum des letzten Jahres und des bisherigen Lebens erfasst wurde. Obwohl dieselbe Unterteilung bereits in diversen Untersuchungen verwendet wurde (z. B. Plöderl und Fartacek 2005, 2009; Wang et al. 2012), bietet sie eher grobe Anhaltspunkte und gibt darüber hinaus keine klaren Informationen zur gegenwärtigen Situation der befragten Personen. 
Auch die Überschneidung der Skalen sozialer Unterstützung und Belastung muss kritisch beurteilt werden. Bereits Fydrich et al. (1999) erläuterten, dass die einzelnen Aspekte sozialer Unterstützung nicht im Sinne unabhängiger Konstrukte interpretiert werden dürfen, was sich auch inhaltlich gut erklären lässt: So kann beispielsweise die Integration in einen Freundeskreis dazu führen, dass die entsprechende Person mehr emotionale und praktische Unterstützung im Alltag wahrnimmt. Die gegenseitige Abhängigkeit der Skalen erschwert allerdings die Interpretation der einzelnen Prädiktoren in einem gemeinsamen Modell, da die Faktoren durch die geteilte Varianz nur schwer trennbar werden. Tatsächlich zeigten sich in unserer Stichprobe etwas höhere Zusammenhänge zwischen den Aspekten sozialer Unterstützung und sozialer Belastung als in der Normierungsstichprobe (Fydrich et al. 1999) und die postulierte Faktorenstruktur konnte nicht repliziert werden. Wie bereits andere Autoren anmerkten (Jäger und Franke 2010), sprechen diese Gründe dafür, die Faktorenstruktur des F-SozU zu überarbeiten. Zudem sollte man diese an einer repräsentativen Stichprobe homo- und bisexueller Personen - idealerweise getrennt - prüfen. Da keine heterosexuelle Vergleichsgruppe befragt wurde, konnte außerdem nicht geklärt werden, ob die gefundenen Zusammenhänge zwischen Aspekten sozialer Unterstützung und der Suizidalität bei heterosexuellen Personen ähnlich stark ausgeprägt sind, oder ob es sich um spezifische Effekte handelt.

Trotz dieser Einschränkungen replizieren die Ergebnisse bisherige Forschungsbefunde und zeigen, dass der Wahrnehmung sozialer Unterstützung und Belastung im Zusammenhang mit der Suizidalität bei homo- und bisexuellen Personen ein großes Gewicht beigemessen werden muss, das auch für die klinische Arbeit und die Suizidalitätsprävention von Bedeutung ist. Der Mangel an Längsschnittuntersuchungen zu Risiko- und Resilienzfaktoren hinsichtlich der Suizidalität von homo- und bisexuellen Personen verdeutlicht den Bedarf weiterer Forschung. Die Zusammenhänge von inhaltlichen Aspekten sozialer Unterstützung und sozialer Belastung mit der Suizidalität müssen weiter untersucht werden, um die zugrundeliegenden Mechanismen besser zu verstehen. Dazu werden weitere Längsschnittuntersuchungen benötigt, die verschiedene Risiko- und Resilienzfaktoren für unterschiedliche Formen der Suizidalität berücksichtigen.

\section{Literatur}

Bailey JM. Homosexuality and Mental Illness. Arch Gen Psychiat 1999; 56: 883 - 884

Botnick MR, Heath KV, Cornelisse PG, Strathdee SA, Martindale SL, Hogg RS. Correlates of Suicide Attempts in an Open Cohort of Young Men who Have Sex with Men. Can J Public Health 2002; 93: $59-62$

Cochand P, Singy P. Développement Identitaire et Risques de Contamination par le VIH chez les Jeunes Homosexuels et Bisexuels en Suisse Romande. Raisons de santé 71. Lausanne: DUPA 2001 [Als Online-Dokument: http://www.iumsp.ch/Publications/pdf/rds71_fr.pdf]

Cohen S, Wills TA. Stress, Social Support, and the Buffering Hypothesis. Psychol Bull 1985; 98 : 310 357

Diamond LM, Lucas S. Sexual-Minority and Heterosexual Youths' Peer Relationships: Experiences, Expectations, and Implications for Well-Being. J Res Adolescence 2004; 14 : 313 - 340 
Diamond GM, Shilo G, Jurgensen E, D'Augelli A, Samarova V, White K. How Depressed and Suicidal Sexual Minority Adolescents Understand the Causes of their Distress. J Gay Lesbian Ment Health 2011; 15: $130-151$

Förtsch G, Penzek C, Röhrle B. Evaluation des Einsamkeits-Bewältigungs-Programms (EBP) in einer psychiatrischen Stichprobe. VPP 2003; 35: 97 - 110

Franke GH, Reimer J, Philipp T, Heemann U. Aspects of Quality of Life through End-Stage Renal Disease. Qual Life Res 2003; 12: 103 - 115

Frei L, Regli D, Widmer A, Znoj H. Angst vor Ablehnung als Risikofaktor für Suizidalität bei homo- und bisexuellen Personen. Sexuologie 2013; 20: 155 - 166

Friedman MS, Marshal MP, Stall R, Cheong J, Wright ER. Gay-Related Development, Early Abuse and Adult Health Outcomes among Gay Males. AIDS Behav 2008; 12: 891 - 902

Fydrich T, Geyer M, Hessel A, Sommer G, Brähler E. Fragebogen zur sozialen Unterstützung (F-SozU): Normierung an einer repräsentativen Stichprobe. Diagnostica 1999; 45: 212 - 216

Fydrich T, Sommer G, Brähler E. F-SozU: Fragebogen zur Sozialen Unterstützung. Göttingen: Hogrefe 2007

Fydrich T, Sommer G, Tydecks S, Brähler E. Fragebogen zur sozialen Unterstützung (F-SozU): Normierung der Kurzform (K-14). Z Med Psychol 2009; 18: 43 - 48

Grob A. Subjective Well-Being and Significant Life-Events across the Life-Span. Swiss J Psychol 1995; 54: $3-18$

Hupcey JE. Clarifying the Social Support Theory - Research Linkage. J Adv Nurs 1998; 27: L1231 1241

Jäger S, Franke GH. Der Fragebogen zur sozialen Unterstützung: Psychometrische Prüfung an einer Stichprobe Studierender. Klin Diagnostik u Evaluation 2010; 3: 427 - 446

Joiner T. Why People Die by Suicide. Cambridge: Harvard University Press 2005

King M, Semlyen J, Tai SS, Killaspy H, Osborn D, Popelyuk D, Nazareth I. A Systematic Review of Mental Disorder, Suicide, and Deliberate Self Harm in Lesbian, Gay, and Bisexual People. BMC Psychiatry 2008; 8: 70

Krampe H, Hautzinger M, Ehrenreich H, Kröner-Herwig B. Depressivität bei Bewohnerinnen und Bewohnern von Altenwohnheimen. Z Kl Psych Psychoth 2003; 32: 117 - 128

Liu RT, Mustanski B. Suicidal Ideation and Self-Harm in Lesbian, Gay, Bisexual, and Transgender Youth. Am J Prev Med 2012; 42: 221 - 228

Marshal MP, Dietz LJ, Friedman MS, Stall R, Smith HA, McGinley J, Thoma BC, Murray PJ, D’Augelli AR, Brent DA. Suicidality and Depression Disparities between Sexual Minority and Heterosexual Youth: a Meta-Analytic Review. J Adolescent Health 2011; 49: 115 - 123

Meyer IH. Prejudice, Social Stress, and Mental Health in Lesbian, Gay, and Bisexual Populations: Conceptual Issues and Research Evidence. Psychol Bull 2003; 129: 674- 697

Meyer IH, Wilson PA. Sampling Lesbian, Gay, and Bisexual Populations. J Couns Psychol 2009; 56: $23-31$

Mustanski B, Liu RT. A Longitudinal Study of Predictors of Suicide Attempts among Lesbian, Gay, Bisexual, and Transgender Youth. Arch Sex Behav 2013; 42: 437 - 448

Nindl A. Einstellung zum Suizid, existentielle Motivation und Suizidalität. Eine vergleichende empirische Studie mit suizidpräventiv relevanten Berufsgruppen und einer klinischen Stichprobe im Rahmen des Projekts: Suizidprävention Salzburg. Salzburg: Unveröffentlichte Dissertation 2001

Paykel G, Myers JK, Lindethal JJ, Tanner J. Suicidal Feelings in the General Population: A Prevalence Study. Brit J Psychiat 1974; 124: 460 - 469

Plöderl M, Fartacek R. Suicidality and Associated Risk Factors among Lesbian, Gay, and Bisexual Compared to Heterosexual Austrian Adults. Suicide Life-Threat 2005; 35: 661 - 670

Plöderl M, Fartacek R. Childhood Gender Nonconformity and Harassment as Predictors of Suicidality among Gay, Lesbian, Bisexual, and Heterosexual Austrians. Arch Sex Behav 2009; 38: 400 - 410

Shilo G, Savaya R. Effects of Family and Friend Support on LGB Youths' Mental Health and Sexual Orientation Milestones. Fam Relat 2011; 60: 318 - 330

Sommer G, Fydrich T. Entwicklung und Überprüfung eines Fragebogens zur sozialen Unterstützung (F-SOZU). Diagnostica 1991; 37: $160-178$

Vinokur AD, Van Ryn M. Social Support and Undermining in Close Relationships: Their Independent Effects on the Mental Health of Unemployed Persons. J Pers Soc Psychol 1993; 65: 350 - 359

Wang J, Häusermann M, Ajdacic-Gross V, Aggleton P, Weiss MG. High Prevalence of Mental Disorders and Comorbidity in the Geneva Gay Men's Health Study. Soc Psych Psych Epid 2007; 42: $414-420$ 
Wang J, Häusermann M, Wydler H, Mohler-Kuo M, Weiss MG. Suicidality and Sexual Orientation among Men in Switzerland: Findings from 3 Probability Surveys. J Psychiatr Res 2012; 46: 980-986

Wethington E, Kessler RC. Perceived Support, Received Support, and Adjustment to Stressful Life Events. J Health Soc Behav 1986; 27: $78-89$

Williams P, Barclay L, Schmied V. Defining Social Support in Context: a Necessary Step in Improving Research, Intervention, and Practice. Qual Health Res 2004; 14: 942 - 960

Korrespondenzadresse

Adrian Widmer

Pfarrhausweg 1

CH-3415 Hasle-Rüegsau

widmer.adrian@students.unibe.ch 